\title{
How to evaluate the value of chest CT in COVID-19: case reports and literature review
}

\author{
Jun Fu ${ }^{1}$ Pian-pian Huang ${ }^{2}$. Qing-dong Yao ${ }^{1}$. Shuang Zhang ${ }^{3} \cdot$ Rui Han $^{1} \cdot$ Hai-feng Liu ${ }^{1}$ Yi Yang ${ }^{1}$. \\ Dong-you Zhang ${ }^{1}$ (1)
}

Received: 27 March 2020 / Revised: 12 July 2020 / Accepted: 8 September 2020 / Published online: 18 September 2020

(c) Springer Nature Singapore Pte Ltd. 2020

\begin{abstract}
The coronavirus disease 2019 (COVID-19) that occurred in Wuhan, Hubei Province, China, has been declared a public health emergency of international concern and a pandemic by the World Health Organization. The Chinese government has temporarily taken strong response measures and effective procedures to stop the further expansion and development of the epidemic. It is important for clinicians to screen, diagnose, and monitor COVID-19.
\end{abstract}

Keywords COVID-19 $\cdot$ Computed tomography $\cdot$ Imaging $\cdot$ SARS-CoV-2

\section{Introduction}

In December 2019, an outbreak of severe acute respiratory syndrome coronavirus 2 (SARS-CoV-2) infection caused the coronavirus disease 2019 (COVID-19) that occurred in Wuhan, Hubei Province, China and spread worldwide in the next few weeks $[1,2]$. Human-to-human transmission of COVID-19 was confirmed by droplets, contact and fomites [3, 4]. The researches have suggested that COVID19 is more contagious than both severe acute respiratory syndrome (SARS) and Middle East respiratory syndrome (MERS) [1, 5-7].

On January 30, 2020, the International Health Regulations Emergency Committee of the WHO declared that the epidemic caused by the new coronavirus had become a "public health emergency of international concern" (PHEIC) [8].

Jun Fu and Pian-pian Huang contributed equally to the article.

Dong-you Zhang

whyyyzdy@163.com

1 Department of Radiology, Wuhan No. 1 Hospital, Zhongshan Avenue \#215, Qiaokou District, Wuhan 430022, Hubei, China

2 Department of Geriatrics, Wuhan No. 1 Hospital, Zhongshan Avenue \#215, Qiaokou District, Wuhan 430022, Hubei, China

3 Department of Clinical Laboratory, Wuhan No.1 Hospital, Zhongshan Avenue \#215, Qiaokou District, Wuhan 430022, Hubei, China
Subsequently, the number of infections in China's neighboring countries (South Korea, Japan) increased exponentially, which caused panic among local people, and the number of new cases reported outside China exceeded the number of new cases in China for the first time on February 26, 2020 [9].

As of on March 16, 2020, there were 81,077 confirmed infections and 3218 deaths in China, and 86,434 confirmed cases with 3388 deaths were reported in 151 countries (Italy, Iran, South Korea, Spain, Germany, were the top five countries) [10]. Therefore, early diagnosis, treatment, and intervention are particularly important. Although viral nucleic acid detection using real-time polymerase chain reaction (RT-PCR) remains the diagnostic criteria, the sensitivity of an initial chest CT was greater than the first RT-PCR [11, 12]. Chest $C T$ can screen patients infected with COVID-19. However, it is worth considering how to evaluate the value and effective use of chest CT in COVID-19. We share three cases of COVID-19 to evaluate the value of chest CT in the epidemic area.

\section{Case 1}

A 44-year-old woman who worked in Wuhan had a history of contact with a COVID-19 patient and presented with a fever of one day and cough for one week on January 19, 2020. Her chest CT (Fig. 1a) findings were abnormal on January 18, 2020. An RT-PCR test upon admission was positive. 


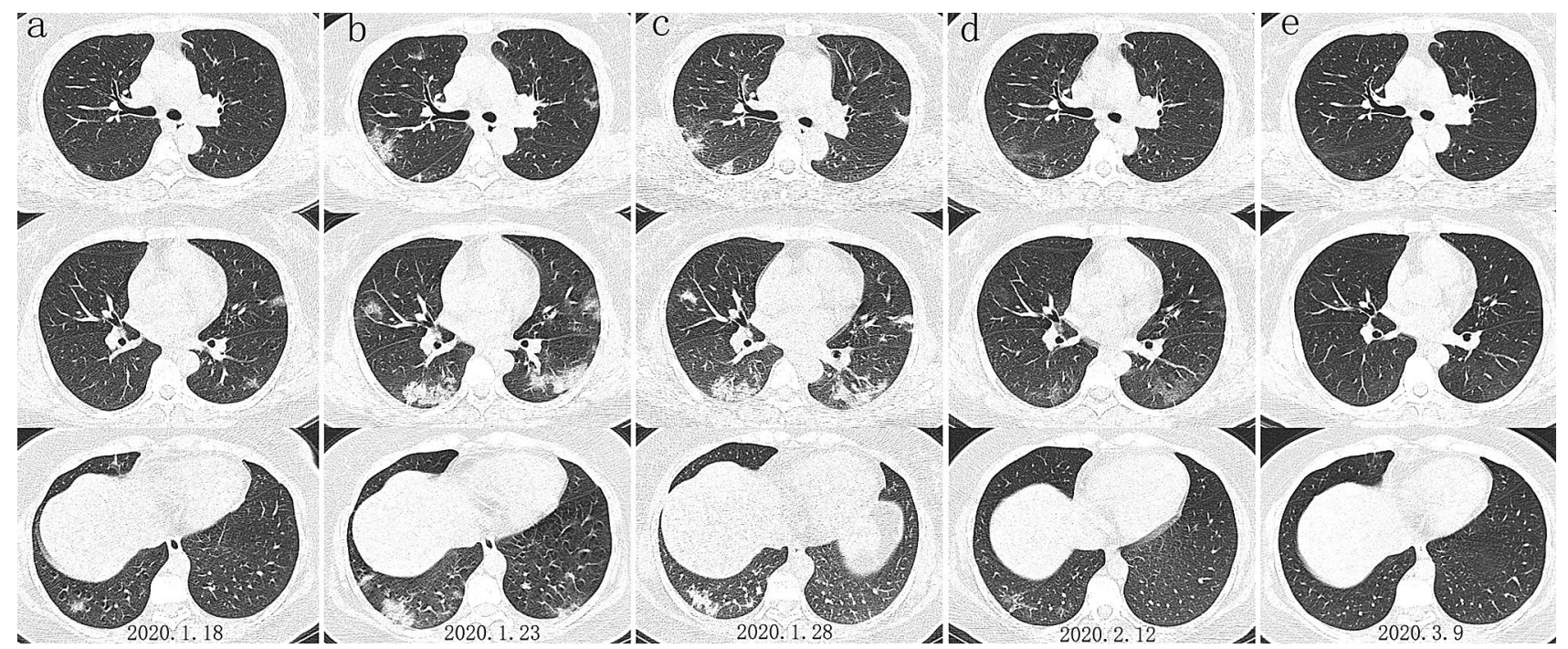

Fig. 1 Chest CT images of a 44-year-old woman. The RT-PCR test was positive. a Axial thin-section chest CT images show minimal ground-glass opacities (GGO) in the subpleural distribution. b, c Images show progressive GGO, mixed GGO and consolidation in the

Laboratory examination showed a normal white blood cell count $\left(5.01 \times 10^{9} / \mathrm{L}\right)$, normal lymphocytes $\left(1.47 \times 10^{9} / \mathrm{L}\right)$, and decreased eosinophils $\left(0.01 \times 10^{9} / \mathrm{L}\right)$. She was diagnosed with COVID-19. A serial follow-up chest CT showed that the lesion was enlarged and eventually absorbed after treatment (Fig. 1b-e).

\section{Case 2}

A 39-year-old woman who worked in Wuhan had a history of contact with a COVID-19 patient and presented with an intermittent cough and expectoration for a week on January 22, 2020. Her chest CT (Fig. 2a) upon admission was normal. Laboratory examination showed a normal white blood cell count $\left(5.08 \times 10^{9} / \mathrm{L}\right)$, normal eosinophils $\left(0.03 \times 10^{9} / \mathrm{L}\right)$, and decreased lymphocytes $\left(0.55 \times 10^{9} / \mathrm{L}\right)$. The patient's chest CT was abnormal and her RT-PCR test was positive after four days of hospitalization. She was diagnosed with COVID-19. A serial follow-up chest CT showed that the lesion was enlarged and partially absorbed after treatment (Fig. 2b-e).

\section{Case 3}

A 38-year-old man who worked in Wuhan had a history of contact with a COVID-19 patient and presented with dizziness and fever for five days on January 30, 2020. A chest CT (Fig. 3a) taken before admission on January 25, 2020, showed abnormalities in the middle lobe of the right bilateral lung. d Images show thinned GGO, indicating absorption in the bilateral lung. e Images show complete absorption and a normal lung

lung. The patient took oral antiviral and anti-infective drugs in self-isolation at home. On January 29, a chest CT showed that the lesion was larger. Upon admission, laboratory examination showed a normal white blood cell count $\left(3.71 \times 10^{9} / \mathrm{L}\right)$, normal lymphocytes $\left(1.71 \times 10^{9} / \mathrm{L}\right)$, normal eosinophils $\left(0.04 \times 10^{9} / \mathrm{L}\right)$, and decreased neutrophils $\left(1.44 \times 10^{9} / \mathrm{L}\right)$. The usual respiratory pathogens (influenza A virus, influenza $B$ virus, respiratory syncytial virus, parainfluenza virus, adenovirus, chlamydia pneumonia, and mycoplasma pneumoniae) were excluded, and he was clinically diagnosed with suspected COVID-19. A serial follow-up chest CT showed that the lesion was enlarged and partially absorbed after treatment (Fig. 3b-d). In the end, COVID-19 was excluded due to three negative RT-PCR tests during hospitalization. Both the RT-PCR test and tests for serum antibodies were negative after discharge.

\section{Discussion}

The outbreak of COVID-19 has changed the lives of the Chinese people. Notably, the Chinese government has temporarily taken strong response measures and implemented effective procedures to stop the further expansion and development of the epidemic. These will serve as a reference for other countries around the world to formulate relevant policies.

In the early stage of the disease outbreak, some suspected patients could not be diagnosed in a timely and effective manner due to the shortage of viral nucleic acid kits, limited sampling methods, and false-negative problems. Most 


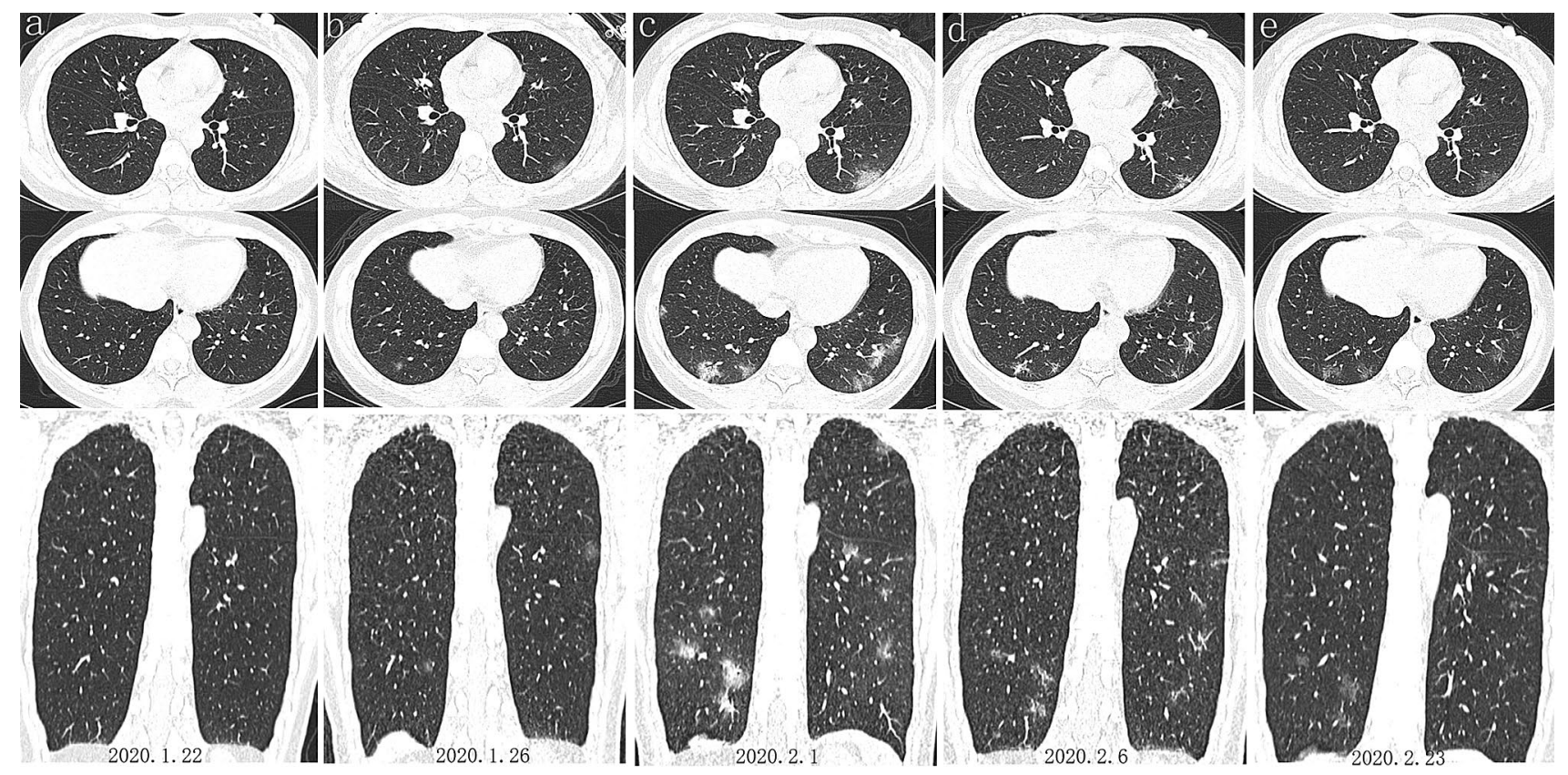

Fig. 2 Chest CT images of a 39-year-old woman. The RT-PCR test was positive. a Axial and coronal images show normal onset. b Images show minimal ground-glass opacities (GGO) in the bilateral lower lung lobes. c Images show progressive mixed GGO and con-

of the recently reported chest CT showed typical radiological features in patients with COVID-19, such as ground glass shadows, multiple lesions, bilateral involvement, lower lobe predilection, subpleural distribution, reversed halo signs, air bronchogram, and crazy-paving patterns [4, 13-16]. At present, the RT-PCR test is the gold standard diagnostic test. However, chest CT can be used as an important complement to RT-PCR for diagnosing COVID-19. In order to control the development and spread of the disease, chest CT, with the characteristics of convenience, rapidness, and intuition, is used to screen and provide an auxiliary diagnostic method in the epidemic area.

With RT-PCR results as a reference in 1014 patients, Ai et al. [11] reported that the sensitivity, specificity, accuracy, positive predictive value, and negative predictive value of chest CT in indicating COVID-19 infection were 97\%, $25 \%, 68 \%, 65 \%$, and $83 \%$, respectively. Almost all patients had initial positive chest CT before or within 6 days of the initial positive RT-PCR results. Clinical diagnostic criteria based on typical chest CT imaging features were temporarily adopted in the revised 5th edition of the Guideline of Diagnosis and Treatment, which was only applicable in Hubei Province, China [17]. This indicates that chest CT can be very useful in screening (early detection of suspected cases) and diagnosing COVID-19 due to the rapid detection and high sensitivity for viral infections and may allow better control of viral spread. solidation in the bilateral lung lobes. d Images show reduced GGO and linear opacities in the bilateral lower lung lobes. e Images show thinned GGO, indicating absorption in the bilateral lower lung lobes

A wide variety of chest CT findings in COVID-19 have been reported in different studies $[18,19]$. The main chest CT feature of COVID-19 pneumonia is the presence of ground-glass opacities (GGO) and consolidation, prominent peripheral subpleural distribution, and posterior part or lower lobe predilection. The GGO may be admixed with areas of focal consolidation and associated with superimposed intralobular reticulations that result in a crazy-paving pattern [20]. The reverse halo sign is frequently observed in patients. These characteristic features can help the epidemic area screen and preliminarily diagnose COVID-19 patients. At the same time, we also need to pay attention to the differential diagnosis. One major differential diagnosis is a pneumonia of bacterial origin. Community-acquired pneumonia is usually characterized by an airspace consolidation in one segment or lobe and the chest CT shows ground glass attenuation, centrilobular nodules, bronchial wall thickening, and mucoid impactions [20]. In the absence of superinfection, COVID-19 pneumonia has no central lobular nodules or mucus impaction on chest CT. SARS, MERS, and other viral pneumonias and non-infectious diseases should be differentiated from COVID-19 based on clinical features and imaging characteristics [21,22].

In addition, chest $\mathrm{CT}$ can be used to monitor the course of the disease and follow the course of COVID-19 patients. Pan et al. [14] summarized the CT imaging changes in COVID19 patients in the early, progressive, peak, and absorption stages. They found that the crazy-paving pattern could be 

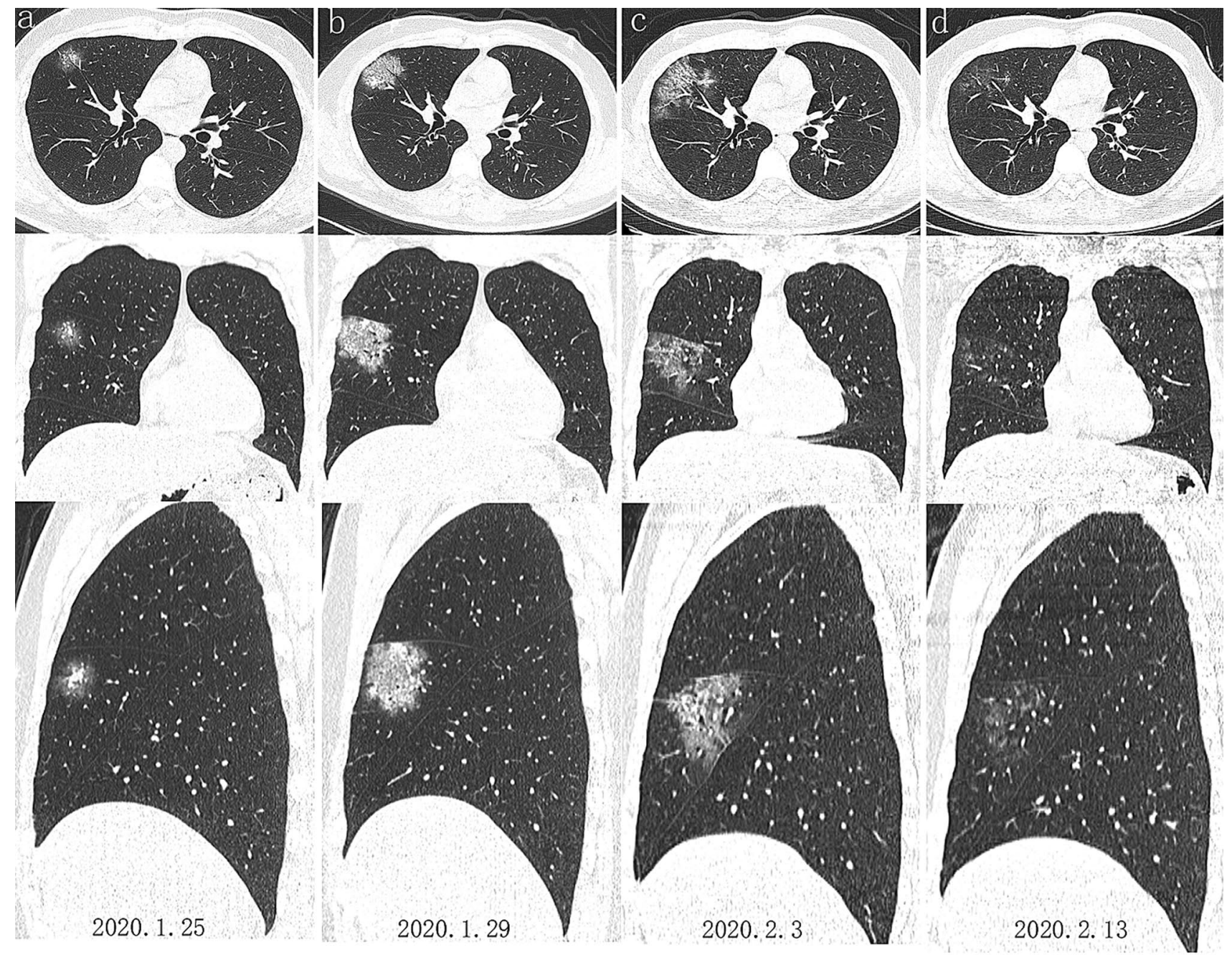

Fig. 3 Chest CT images of a 38-year-old man. The RT-PCR test was negative. a Axial, coronal, and sagittal images show a solitary, rounded, peripheral ground-glass opacity (GGO) with halo sign and air bronchial sign in the middle lobe of the right lung. $\mathbf{b}$ Images show progressive mixed GGO and consolidation in the middle lobe of the

right lung. $\mathbf{c}$ Images show increased GGO and linear opacities in the middle lobe of the right lung. d Images show reduced GGO and linear opacities, indicating absorption in the middle lobe of the right lung

used as an indicator of the course of the disease. Shi et al. [23] indicated that the radiological evolution of COVID-19 was consistent with the clinical course, and revealed that the specific signs in chest $\mathrm{CT}$ could effectively reflect the progress and outcome of the disease.

The three cases above showed that chest CT could effectively screen, diagnose, and monitor COVID-19. Most studies reported that the majority of patients infected with SARS-CoV-2 were positive in both the chest CT and RTPCR test (Case 1), and chest CT could assess the severity of lung damage, which was helpful for clinical treatment. However, there were some false negatives (Case 2) and false positives (Case 3 ) in chest CT in diagnosing COVID19 based on positive RT-PCR results. The reason for the false negatives in chest CT may be that the virus is located

in the upper respiratory tract in the early stage and does not cause abnormal changes in the lower respiratory tract and lungs. The patients might also be in the incubation period [24]. This suggests that a normal chest CT cannot exclude a diagnosis of COVID-19, especially for patients with early onset of symptoms. The false positives in chest CT maybe due to the CT imaging findings of COVID-19 were similar to other viral pneumonia. However, some "false-positive" cases on CT may indeed be "true-positive" for the relatively low rate of positive RT-PCR results [11]. Hao reported a clinically suspected COVID-19 patient with an abnormal chest CT. The patient was not diagnosed until the fourth RT-PCR test was positive [25]. We suggest that RT-PCR tests should be repeated, especially for patients with a high clinical suspicion of COVID-19. 


\section{Conclusion}

Chest CT plays an important role in the screening, diagnosis, evaluation, and monitoring of COVID-19. The combination of a patient's history, clinical manifestations, typical CT imaging features, laboratory findings, and RT-PCR test results will be helpful for the diagnosis of COVID-19.

Funding We declare that we have not received any financial assistance.

\section{Compliance with ethical standards}

Conflict of interest We declare that there is no conflict of interest for this publication.

\section{References}

1. Huang C, Wang Y, Li X, et al. Clinical features of patients infected with 2019 novel coronavirus in Wuhan, China. Lancet. 2020;395(10223):497-506. https://doi.org/10.1016/S0140 $-6736(20) 30183-5$.

2. Zhu N, Zhang D, Wang W, et al. A novel coronavirus from patients with pneumonia in China, 2019. N Engl J Med. 2020;382(8):72733. https://doi.org/10.1056/NEJMoa2001017.

3. Zhou P, Yang XL, Wang XG, et al. A pneumonia outbreak associated with a new coronavirus of probable bat origin. Nature. 2020;579(7798):270-3. https://doi.org/10.1038/s4158 6-020-2012-7.

4. Zu ZY, Jiang MD, Xu PP, Disease C, et al. (COVID-19): a perspective from China. Radiology. 2019;2020:200490. https://doi. org/10.1148/radiol.2020200490.

5. Chan JF, Yuan S, Kok KH, et al. A familial cluster of pneumonia associated with the 2019 novel coronavirus indicating person-to-person transmission: a study of a family cluster. Lancet. 2020;395(10223):514-23. https://doi.org/10.1016/S0140 $-6736(20) 30154-9$.

6. Munster VJ, Koopmans M, van Doremalen N, et al. A novel coronavirus emerging in China-key questions for impact assessment. N Engl J Med. 2020;382(8):692-4. https://doi.org/10.1056/ NEJMp2000929.

7. Wang C, Horby PW, Hayden FG, et al. A novel coronavirus outbreak of global health concern. Lancet. 2020;395(10223):470-3. https://doi.org/10.1016/S0140-6736(20)30185-9.

8. World Health Organization. Statement on the second meeting of the International Health Regulations (2005) Emergency Committee regarding the outbreak of novel coronavirus $(2019-\mathrm{nCoV})$. 2020. https://www.who.int/news-room/detail/30-01-2020-state ment-on-the-second-meeting-of-the-international-health-regul ations-(2005)-emergency-committee-regarding-the-outbreak-ofnovel-coronavirus-(2019-ncov).

9. World Health Organization. WHO Director-General's opening remarks at the mission briefing on COVID-19-26 February 2020. 2020. https://www.who.int/dg/speeches/detail/who-direc tor-general-s-opening-remarks-at-the-mission-briefing-on-covid -19---26-february-2020.

10. World Health Organization. Coronavirus disease 2019 (COVID19) Situation Report-56. 2020. https://www.who.int/docs/defau 1t-source/coronaviruse/situation-reports/20200316-sitrep-56-covid -19.pdf?sfvrsn $=9 \mathrm{fda} 7 \mathrm{db} 22$.

11. Ai T, Yang Z, Hou H, et al. Correlation of chest CT and RTPCR testing in coronavirus disease 2019 (COVID-19) in China: a report of 1014 cases. Radiology. 2020;2020:200642. https://doi. org/10.1148/radiol.2020200642.

12. Fang Y, Zhang H, Xie J, et al. Sensitivity of chest CT for COVID-19: comparison to RT-PCR. Radiology. 2020. https:// doi.org/10.1148/radiol.2020200432.

13. Chung M, Bernheim A, Mei X, et al. CT imaging features of 2019 novel coronavirus (2019-nCoV). Radiology. 2020;295(1):202-7. https://doi.org/10.1148/radiol.2020200230.

14. Pan F, Ye T, Sun P, et al. Time course of lung changes at chest CT during recovery from coronavirus disease 2019 (COVID-19). Radiology. 2020;295(3):715-21. https://doi.org/10.1148/radio 1.2020200370.

15. Pan $\mathrm{Y}$, Guan $\mathrm{H}$, Zhou S, et al. Initial CT findings and temporal changes in patients with the novel coronavirus pneumonia (2019nCoV): a study of 63 patients in Wuhan, China. Eur Radiol. 2020;30(6):3306-9. https://doi.org/10.1007/s00330-020-06731-x.

16. Song F, Shi N, Shan F, et al. Emerging 2019 novel coronavirus (2019-nCoV) pneumonia. Radiology. 2020;295(1):210-7. https:// doi.org/10.1148/radiol.2020200274.

17. General Office of National Health Committee. Notice on the issuance of a program for the diagnosis and treatment of novel coronavirus (2019-nCoV) infected pneumonia (trial revised fifth edition). 2020. https://www.nhc.gov.cn/yzygj/s7653p/202002/ d4b895337e19445f8d728fcaf1e3e13a.shtml.

18. Cheng Z, Lu Y, Cao Q, et al. Clinical features and chest CT manifestations of coronavirus disease 2019 (COVID-19) in a single-center study in Shanghai, China. AJR Am J Roentgenol. 2019;2020:1-6. https://doi.org/10.2214/AJR.20.22959.

19. Salehi S, Abedi A, Balakrishnan S, et al. Coronavirus disease 2019 (COVID-19): a systematic review of imaging findings in 919 patients. AJR Am J Roentgenol. 2020;2020:1-7. https://doi. org/10.2214/AJR.20.23034.

20. Hani C, Trieu NH, Saab I, et al. COVID-19 pneumonia: a review of typical CT findings and differential diagnosis. Diagn Interv Imaging. 2020;101(5):263-8. https://doi.org/10.1016/j. diii.2020.03.014.

21. Fan L, Li D, Xue H, et al. Progress and prospect on imaging diagnosis of COVID-19. Chin J Acad Radiol. 2020. https://doi. org/10.1007/s42058-020-00031-5.

22. Li B, Li X, Wang Y, et al. Diagnostic value and key features of computed tomography in Coronavirus Disease 2019. Emerg Microbes Infect. 2020;9(1):787-93. https://doi.org/10.1080/22221 751.2020 .1750307 .

23. Shi H, Han X, Jiang N, et al. Radiological findings from 81 patients with COVID-19 pneumonia in Wuhan, China: a descriptive study. Lancet Infect Dis. 2020;20(4):425-34. https://doi. org/10.1016/S1473-3099(20)30086-4.

24. Guan WJ, Ni ZY, Hu Y, et al. Clinical characteristics of coronavirus disease 2019 in China. N Engl J Med. 2020;382(18):1708-20. https://doi.org/10.1056/NEJMoa2002032.

25. Hao W, Li M. Clinical features of atypical 2019 novel coronavirus pneumonia with an initially negative RT-PCR assay. J Infect. 2020;80(6):671-93. https://doi.org/10.1016/j.jinf.2020.02.008.

Publisher's Note Springer Nature remains neutral with regard to jurisdictional claims in published maps and institutional affiliations. 